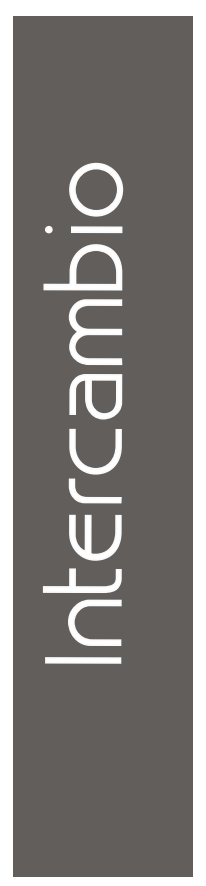

\title{
ESTADO DEL ARTE EN EL DISEÑO DE LAGUNAS DE ESTABILIZACIÓN
}

Armando Cubillos Z.*

* Profesor Instituto CIDIAT - Universidad de Los Andes - Mérida - Venezuela

\section{RESUMEN}

El autor muestra el tratamiento de aguas residuales municipales y de industrias en lagunas de potabilización como una alternativa viable y económica. Hace además una completa descripción de los tipos de lagunas y modelos existentes. Mos presenta también el proceso de diseño de las estructuras y su operación y mantenimiento, finalizando con un ejercicio práctico. 


\section{ABSTRACT}

This paper presents the municipal and industrial wasterwater treatment as an economic and possible alternative. The work not only intends so show a complete description of the types and models of lakes, but also it presents the design process of structures, operation, and maintenance. Finally, presents a practical exercise.

\section{INTRODUCCIÓN}

El tratamiento de aguas residuales municipales o de industrias en lagunas de estabilización constituye una alternativa viable donde existan terrenos disponibles de bajo costo y este método de tratamiento sea más económico que otros métodos alternos, tales como: lodos activados, digestión aerobia, biodiscos, filtros biológicos, digestión anaerobia o la unidad anaerobia de flujo ascendente (UASB ó RAFA). De estos sistemas sólo el UASB compite en costo con las lagunas de estabilización.

Oswald, 1995, anota que debido a la eficiencia de las lagunas, "cree que en este mundo de explosión de la población y agotamiento de recursos, las lagunas deben continuar siendo una parte creciente en nuestro manejo de desechos líquidos". Además, teniendo en cuenta que las lagunas son eficaces en la remoción de organismos patógenos en el trópico, se han hecho avances significativos en el uso del efluente de lagunas en riego de cultivos y cría de peces, como un método eficaz de utilización de los fertilizantes y la materia orgánica remanente en las aguas tratadas.

El diseño de lagunas de estabilización se ha hecho utilizando modelos desarrollados de información obtenida en lagunas que operan en diferentes condiciones meteorológicas y de mezcla a las del sitio de las lagunas. Esta circunstancia hace necesario tener extremo cuidado en que el método seleccionado corresponda a condiciones meteorológicas semejantes para reducir los riesgos inherentes al diseño, no se debe utilizar modelos desarrollados para la zona templada o fría en el trópico y subtrópico, donde la temperatura ambiente no tiene variaciones estacionales.

Los modelos existentes permiten estimar la eficiencia de la laguna en la remoción de DBO, DQO, coliformes, calcular el área, volumen, período de retención y estimar la DBO y MMP en el efluente y establecer las dimensiones y el número de lagunas necesarias en paralelo y en serie para obtener el grado de tratamiento exigido.

Sin embargo, existen vacíos de información que deben ser llenados en trabajos de investigación e innovaciones que vendrán para atender a la solución de problemas específicos de uso del efluente de lagunas con el propósito de hacer un mejor uso de los recursos y establecer las bases del desarrollo sostenible. Las investigaciones más recientes se orientan a aprovechar el contenido de materia orgánica y fertilizantes en el efluente tratado en riego de cultivos, piscicultura y producción de forraje para animales, con el propósito de disminuir el vertido de estos poluentes a cuerpos de agua, proteger el ambiente y aumentar la producción de alimentos para una población cada vez más numerosa.

\section{TIPOS DE LAGUNAS Y DIAGRAMAS COMUNES DE FLUJO}

Tradicionalmente se han distinguido cuatro tipos de lagunas: aerobias, anaerobias, facultativas y de maduración. Actualmente, Pearson, 1995, distingue cerca de 10 tipos de lagunas. En este trabajo no se hace referencia a las lagunas aireadas y se presenta una definición breve de los tipos básicos de lagunas, teniendo en cuenta que de ellos se desarrollaron tipos nuevos y más eficientes de lagunas. 
Una laguna es un reactor diseñado y construido mediante excavación del suelo y construcción de diques para formar vasos capaces de retener el agua residual un período determinado de tiempo para obtener un efluente de una calidad dada.

Laguna aerobia es aquella en la cual hay oxígeno disuelto en toda la masa de agua, tienen poca profundidad, hasta donde penetra la luz solar, generalmente 0,50 metros.

La laguna anaerobia es profunda, de 2,50 a 5,00 $m$, recibe una carga orgánica alta, 100 a 400 $\mathrm{g} / \mathrm{m}^{3} \mathrm{~d}$. Mo hay oxígeno disuelto, se forma una capa de material flotante que la aísla del aire y bien operada no debe producir malos olores, generalmente, recibe efluentes concentrados.

La laguna facultativa tiene una profundidad de 1,80 a 2,20 m, la capa profunda es anaerobia y la capa superficial es aerobia, en el trópico acepta cargas superficiales entre 250 y 600 kg DBO/ha.d.. Es el tipo de laguna de estabilización más comúnmente usado.

La laguna de maduración recibe el efluente de otros sistemas de tratamiento o de lagunas facultativas, se utiliza para destruir organismos patógenos, su profundidad máxima es de 1,50 $\mathrm{m}$.

Un sistema de lagunas consiste en un conjunto de varios tipos de lagunas en serie, de manera que el efluente satisfaga las exigencias de la agencia responsable del control de la calidad de agua o del control de la polución. La Figura 1 muestra dos esquemas o diagramas de flujo de entre muchos que son posibles.

\section{MODELOS O ECUACIONES PARA EL DISEÑO}

En este aparte se presentan y discuten los modelos más conocidos para dimensionar lagunas de estabilización en función de la la remoción de materia orgánica, medida como DBO y la extinción de coliformes medidos como MMP/100 ml. Se omite la presentación de algunos modelos usados en la zona templada, debido a que no son totalmente aplicables a las condiciones ambientales del trópico:

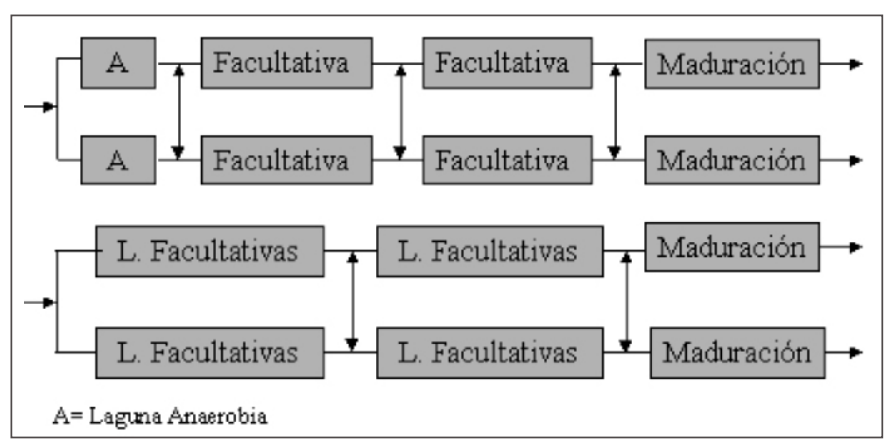

Figura 1. Diagramas de Flujo de Lagunas de Estabilización

\section{Modelo de Hemann y Gloyna}

Hermann y Gloyna (1958) de resultados de experimentos desarrollados a $35{ }^{\circ} \mathrm{C}$, para una remoción de 90 por ciento de la DBO, propusieron una expresión para el cálculo del volumen de la laguna a escala real, así:

$$
V=7 Q_{a} *\left(S_{a} / 200\right) * 1,085^{(35-T)}(1)
$$

Donde :

$\mathrm{V}=$ Volumen de la laguna en $\mathrm{m}^{3}$

$Q_{a}=$ Aporte de aguas residuales a la laguna en $\mathrm{m}^{3} / \mathrm{d}$

$\mathrm{S}_{\mathrm{a}}=\mathrm{DBO}_{5}$ en las aguas residuales, $\mathrm{mg} / \mathrm{Ló} \mathrm{g} / \mathrm{m}^{3}$,

$\mathrm{T}=$ Temperatura media del agua durante el mes más frío del año.

Un análisis de regresión de datos experimentales de las lagunas experimentales del ICA y a escala real del CIAT, con remociones entre el 60 y 90 por ciento, produjo la Ec. 2 con un coeficiente de correlación de 0,995, para estimar el volumen de lagunas facultativas en el trópico:

$$
V=2,8 Q_{a} *\left(S_{a} / 200\right) * 1,085^{(T-35)}
$$


Esta ecuación se puede modificar dividiendo ambos términos por el área de la laguna y teniendo en cuenta que $\left(Q_{a} S_{a} / A\right)=C S_{a}$ y se obtiene una expresión para estimar la carga superficial máxima que puede aceptar la laguna facultativa, así:

$$
C S_{a}=714,286 h 1,085^{(T-35)}
$$

Donde $: \mathrm{h}=$ profundidad de la laguna, $\mathrm{m}$

En un documento elaborado para el diseño de lagunas en el mediterráneo, Pescod (1980) propone la siguiente ecuación para estimar la carga superficial máxima en lagunas facultativas, así:

$$
C S_{a}=60,3 * 1,099^{T a}
$$

Donde : $T_{a}=$ temperatura ambiente, ${ }^{\circ} \mathrm{C}$

Estas dos ecuaciones producen resultados semejantes o del mismo orden de magnitud.

\section{Modelo de Oswald y Gotaas}

Este modelo fue propuesto para lagunas aerobias (Oswald y Gotaas, 1957). El oxígeno sintetizado es función de la radiación solar y la densidad de algas, la carga orgánica se fija en función de la síntesis de oxígeno. Mo se presentan las expresiones propuestas por los autores del modelo teniendo en cuenta que no es muy usado en el trópico.

\section{Modelo de Mezcla Completa o de Marais}

El modelo se basa en el supuesto de que en lagunas facultativas hay mezcla completa y cinética de primer orden, (Marais, 1966). La expresión básica es la siguiente:

$$
S=\frac{S a}{(1+K * P R)}(5)
$$

Donde : $\mathrm{Sa}=\mathrm{DBO}_{5}$ en el afluente, $\mathrm{g} / \mathrm{m}^{3}$,

$\mathrm{S}=\mathrm{DBO}_{5}$ soluble en el efluente, $\mathrm{g} / \mathrm{m}^{3}$

$\mathrm{K}=$ Constante de gradación de la DBOen la laguna a temperatura local, 1/d.

$\mathrm{PR}=$ Período de retención hidráulico en la laguna, días.
En este modelo es difícil evaluar la constante de degradación, K, Yanez (1980) propone una expresión para estimar el valor de $K$, es la siguiente:

$$
K=\frac{P R}{-14,77+4,64 P R}(6)
$$

Donde : $P R=$ período de retención hasta la laguna en consideración para lagunas en serie.

También es necesario corregir la constante de degradación, K, para la temperatura del agua en la laguna, debido a que, generalmente, se obtiene un valor para $20^{\circ} \mathrm{C}$ en el laboratorio, se usa la siguiente expresión:

$$
K_{T}=K_{20} * \Theta^{(T-20)}(7)
$$

$\Theta=$ Cons $\tan$ teparacorregirefectodelatemperatura,iguala 1,085

En este modelo se supone que hay mezcla completa en la laguna, en realidad no hay mezcla completa, debido a esto existen objeciones sobre la validez del modelo y se han propuesto modelos para una combinación de flujo a pistón y mezcla parcial con dispersión (Thirumurthy, 1974), el cual será tratado adelante.

\section{Correlaciones o Modelos Estadísticos}

Del análisis estadístico de resultados de la operación de lagunas facultativas para 143 condiciones diferentes, con eficiencias entre el 70 y 90 por ciento y cargas orgánicas entre 50 y 500 Kg DBO/ha.día, Mc Garry y Pescod (1970) desarrollaron un modelo en base a una expresión de la forma:

$$
C S_{r}=A+B * C S_{a}(8)
$$

Donde : $\mathrm{CS}_{\mathrm{a}}=$ Carga superficial aplicada a la laguna, kg DBO/ha.d.

$\mathrm{CS}_{\mathrm{r}}=$ Carga superficial removida en la laguna, Kg DBO/ha.d

$A=$ Factor numérico, resultado del análisis estadístico de datos de La operación.

$B=$ Coeficiente numérico, resultado del análisis estadístico. 
Este modelo ha sido objeto de la atención de varios autores, quienes han desarrollado expresiones para diseñar lagunas facultativas, a continuación se presentan las ecuaciones propuestas y el nombre del autor:

$$
\begin{gathered}
C S_{r}=10,35+0,725 * C S_{a} \text { McGarryyPescod }(1970) \\
C S_{r}=7,67+0,8063 * C S_{a} \text { Yanez }(1980) \\
C S_{r}=2,0+0.79 * C S_{a} \quad \text { MaraySilva }(1969) \\
C S_{r}=19,4+0,586 * C S_{a} \quad \text { Kawai }(\text { CETESB })(1981) \\
C S_{r}=25,13+0,675 * C S_{a} \quad \text { Cubillos }(1984)
\end{gathered}
$$

\section{Lagunas Secundarias}

Es frecuente el uso de lagunas en serie, en esta caso a las lagunas facultativas les siguen lagunas facultativas secundarias para remover más DBO. En este trabajo se incluyen dos ecuaciones desarrolladas para estimar la carga superficial removida, ellas son:

$$
\begin{aligned}
& C S_{r}=0,765 * C S_{a}-0,80 \text { Yanez }(1981) \\
& C S_{r}=0,8167 * C S_{a}-3,82 \text { Cubillos }(1984)
\end{aligned}
$$

La carga superficial máxima que puede recibir una laguna facultativa secundaria se fija de la experiencia, no existe una expresión propuesta por investigadores. Se pudiera utilizar la misma carga de la laguna primaria, Ecuaciones 3 ó 4 de este trabajo, y verificar su validez en lagunas experimentales. Las cargas superficiales aplicadas más comunes en lagunas secundarias son del orden de 300 a 450 Kg DBO / ha.d.

\section{Lagunas Anaerobias}

Las lagunas anaerobias se diseñan mediante expresiones empíricas o fijando una carga orgánica por unidad de volumen. Mumerosos autores están de acuerdo en que las lagunas anaerobias operan satisfactoriamente con cargas orgánicas entre 100 y $400 \mathrm{gDBO} / \mathrm{m}^{3} \mathrm{~d}$. y remociones entre el 50 y 80 por ciento, en el tratamiento de aguas residuales municipales y cargas entre 100 y $200 \mathrm{gDBO} / \mathrm{m}^{3} \mathrm{~d}$ producen remociones del orden del 65 por ciento.
Vincent en 1963 propuso para Zambia, en condiciones de mezcla completa y temperatura del agua de $20{ }^{\circ} \mathrm{C}$, en Romero (1995), la expresión empírica:

$$
S_{e}=\frac{S_{a}}{\left(\frac{S_{e}}{S_{a}}\right)^{n} * K * P R+1}
$$

Donde $: n=4,8$

$$
k=6,0
$$

Sa y $\mathrm{Se}=\mathrm{DBO}$ en el afluente y efluente respectivamente.

En esta ecuación, se calcula por aproximaciones, teniendo en cuenta que es aproximadamente igual a 0,5 Sa.

\section{Modelos para la Remoción de Coliformes}

Se sabe que las lagunas de estabilización son más eficientes en la remoción de organismos patógenos, tales como: helmintos, protozoarios y bacterias, que otros sistemas de tratamiento de aguas residuales. Esta realidad hace que el tratamiento de aguas residuales en lagunas sea de interés en el trópico donde existe una alta incidencia de enfermedades de origen hídrico. Se han propuesto varios modelos para estimar la remoción de coliformes en lagunas de estabilización, los más conocidos son: a) el modelo para un reactor de mezcla completa y cinética de primer orden de Marais (1974) y, b) el modelo para mezcla no ideal con dispersión, según el modelo desarrollado por Whener $y$ Wilhem (1958), ampliado por Thirumurthi (1969) y complementado por Polprasert y Bhattarai (1985).

La eficiencia de las lagunas de estabilización para remover materia orgánica y destruir organismos patógenos depende de las transformaciones bioquímicas y del modelo de flujo, además de otros factores ambientales y climatológicos como: la radiación solar, la lluvia, el viento y la temperatura. La ocurrencia de altos valores del pH, la producción de compuestos tóxicos extracelulares por las algas, la exposición a la luz ultravioleta y la disminución de nutrientes 
contribuyen a la extinción de bacterias (Polprasert, 1985). Los huevos de helmintos y quistes de amebas separan por sedimentación.

\section{Modelo de Mezcla Completa}

Marais (1974) supuso que una laguna de estabilización se comporta como un reactor completamente mezclado y propuso la siguiente expresión para estimar el MMP de coliformes en el efluente de una laguna:

$$
N=\frac{N_{0}}{1+K * P R}(11)
$$

Donde :

$M=$ Densidad de coliformes en el efluente, $\mathrm{MMP} / 100 \mathrm{ml}$

$M_{0}=$ D ensidad de coliformes en el afluente, $\mathrm{MMP} / 100 \mathrm{ml}$.

$\mathrm{K}=$ Constante de extinción bacteriana a la temperatura de la laguna, $1 / d$

$\mathrm{PR}=$ Período de retención hidráulico, días

En el caso en que existan varias lagunas facultativas y de maduración en serie, la ecuación 11 toma la forma general:

$$
N_{i}=\frac{N_{0}}{\left(1+K_{1} * P R_{1}\right) *\left(\left(1+K_{2} * P R_{2}\right) * \ldots *\left(1+K_{i} * P R_{i}\right)\right.}(12)
$$

Marais (1974) encontró que la constante de extinción, $K_{20}\left(a 20{ }^{\circ} \mathrm{C}\right.$ ) es iguala $2,6 / d$, es necesario hacer una corrección por temperatura $\mathrm{aK}_{20}$

$$
K_{T}=K_{20} * \Theta^{(T-20)}(13)
$$

Donde : $\Theta=$ Factor de corrección en la extinción de bacterias, igual a 1,19 (Marais, 1974). Otros autores, entre ellos Yanez, 1993, proponen un valor de 1,07

Cuando existe un número infinito de lagunas en serie, el flujo se asemeja al que ocurre en un reactor de flujo a pistón o a un río y la extinción bacteriana se describe en la Ecuación 14:

$$
N=N_{0} * \mathrm{E}^{-K * P R}
$$

\section{Modelo de Mezcla Parcial con Dispersión}

Polprasert y Bhattarai (1985) proponen un modelo para lagunas facultativas y de maduración, suponiendo flujo no ideal con dispersión para estimar la eficiencia en la remoción de DBO y coliformes según la Ecuación propuesta por Wehner y Wilhem (1956).

$$
\frac{C}{C_{0}} O \frac{N}{N_{0}}=\frac{4 a * \mathrm{E}^{(1 / 2 d)}}{(1+a)^{2} * \mathrm{E}^{(a / 2 d)}-(1-a)^{2} * \mathrm{E}^{(-a / 2 d)}}
$$

Donde : $\quad a=\sqrt{1+4 K * P R * d}$

C ó $M=$ Concentración de DBO ó MMP de coliformes en el efluente.

$C_{0}$ ó $M_{0}=$ DBO ó MMP de coliformes en el afluente.

$K=\quad$ Coeficiente de reacción para DBO o Extinción de bacterias coliformes.

$\mathrm{d}=\quad$ Múmero de dispersión del flujo. $d=\frac{D}{U * L}$

$D=\quad$ Dispersión longitudinal o axial del flujo.

$\mathrm{L}=\quad$ Longitud del recorrido del flujo entre la entrada y la salida de la laguna.

La Ecuación 15 se puede simplificar en todos los casos en los cuales el valor de, d, sea menor a 2 , tal es el caso en lagunas de estabilización, entonces según Thirumurthi (1969) resulta la Ecuación 16, así:

$$
\frac{N}{N_{0}}=\frac{4 a * \mathrm{E}^{[(1-a) / 2 d]}}{(1+a)^{2}}(16)
$$

El factor de dispersión, d, se puede determinar en función del período de retención, las dimensiones de la laguna y la viscosidad del agua, según Polprasert y Bhatharai (1985):

$$
d=\frac{0,184[\operatorname{PR} \vee(W+2 Z)]^{0,489}(W)^{1,511}}{(L Z)^{1,489}}
$$

Ahora falta encontrar una expresión para estimar el valor de la constante de extinción bacteriana en la laguna de estabilización, $K_{b}$. León y Moscoso, (1996), de información obtenida en las lagunas de estabilización de San Juan, proponen expresiones para calcular $K_{b}$ en lagunas 
primarias, secundarias y terciarias, así:

Lagunas primarias: $K_{b}=0,477 * 1,18^{(T-20)}(18)$

Lagunas secundarias: $K_{b}=0,904 * 1,04^{(T-20)}(19)$

Lagunas Terciarias: $K_{b}=0,811 * 1,09^{(T-20)}(20)$

La constante de extinción para lagunas terciarias, $K_{b}$, es el resultado del análisis estadístico de sólo tres datos. Se debe tener en cuenta que sólo indica un estimado del orden de magnitud y tendencia (León y Moscoso, 1996). En estos casos es conveniente utilizar el modelo de mezcla completa y comparar los resultados, para su interpretación.

Von Sperling (1999) hace una revisión los valores reportados en la literatura de la constante de extinción bacterial, $K_{b}$, en lagunas de estabilización, encuentra una gran dispersión de los ordenes de magnitud para mezcla completa y menor para flujo a pistón y flujo disperso. Este autor hace un cálculo de las constantes de extinción para 33 lagunas en el trópico y subtrópico para mezcla completa y para flujo disperso según Yanez (1993) y Agunwamba et al (1992). Los valores de $K_{b}$ para flujo disperso, encontrados por Von Sperling, usando las expresiones de Yanez y Agunwamba son semejantes para profundidades de las lagunas entre 1,40 y 2,40 metros.

La expresión propuesta por Yanez (1993) para el cálculo del número de dispersión del flujo, d, es más simple y aparece como la ecuación 21 .

$$
d=\frac{(L / B)}{-0,26+0,254(L / B)+1,014(L / B)^{2}}
$$

Von Sperling hacen un análisis de regresión y obtiene una expresión para el cálculo de $K_{b}$ en lagunas con flujo disperso, la cual se toma como ecuación 22.

$$
K_{b}=0,917 H^{-0,877}(P R)^{-0,329}
$$

\section{PROCESO DE DISEÑO}

El diseño de un sistema de lagunas de estabilización es un proceso complejo, a pesar de la simplicidad, debido a que tiene carácter multidisciplinario y se debe tener extremo cuidado en seleccionar un modelo para establecer las dimensiones de las lagunas, aplicable a las condiciones climáticas del lugar. Además exige de un conocimiento de los procesos de remoción de poluentes. Se presenta una síntesis de las actividades a desarrollar durante el diseño:

* Selección del sitio según la: topografía, permeabilidad del suelo, capacidad de soporte, vulnerabilidad a desastres, distancia al centro urbano, viviendas y dirección del viento.

* Proyecciones de población para el período de diseño.

* Estimado de flujos actuales y futuros de aguas residuales.

* Características de las aguas residuales, medida de: temperatura, DBO, DQO, Sólidos, Mitrógeno, Fósforo, grasas y MMP de coliformes, etc.

* Estimado de las cargas orgánicas actuales y futuras a ser llevadas al sistema de lagunas.

* Selección del modelo a ser usado en el diseño de las lagunas y selección del diagrama de flujo, con o sin lagunas anaerobias.

* Si hay laguna anaerobia, se debe calcular el volumen fijando la carga orgánica por unidad de volumen: 100 a $400 \mathrm{~g} / \mathrm{m}^{3}$

* Cálculo de la carga superficial máxima que puede procesar la laguna primaria facultativa según la Ec. 3, ó 4.

* Cálculo del área de la laguna facultativa según la definición de carga superficial.

* Cálculo del volumen, para una profundidad dada.

* Cálculo de las pérdidas por infiltración y evaporación.

* Cálculo del período de retención, tomando en cuenta las pérdidas por evaporación e infiltración. $P R=2 * V /\left(Q_{a}+Q_{e}\right)$ 
Qa y Qe = Caudal afluente y efluente respectivamente, $\mathrm{m}^{3} / \mathrm{d}$ )

* Se dan dimensiones a la laguna o lagunas.

* Cálculo de la carga superficial removida, CS $_{\text {r }}$ de la Ecuación 8.

* Cálculo de la DBO en el efluente de la carga superficial que sale de la laguna (CSe = CSa CSr) y la definición de carga superficial: $\mathrm{CSe}=\mathrm{Qe}$ BDOe/A

* Cálculo del MMP de coliformes según la EC. 10 ó 15.

* Comparación de la DBO y el MMP estimados en el efluente de la laguna y las normas de calidad de agua para efluentes. Si no se satisface la norma es indispensable diseñar más lagunas en serie, siguiendo el mismo proceso de cálculo y teniendo cuidado de utilizar las ecuaciones aplicables en cada caso.

En el proceso de diseño es conveniente verificar las condiciones de operación del sistema de lagunas en el tiempo durante el período de diseño, de manera que sea posible proponer ampliaciones periódicas en función del aumento estimado de la carga orgánica, evitar que las lagunas operen con cargas muy bajas o demasiado altas y no satisfagan las exigencias prevista en el diseño para la calidad del efluente.

Finalmente, se han hecho investigaciones sobre el efecto de construir deflectores para reducir los espacios muertos y corto circuitos, mejorar el régimen de flujo y la eficiencia de las lagunas. Kilani y Ogunrombi (1988) en modelos de lagunas, encontraron que la eficiencia en la remoción de DBO, DQO y sólidos aumenta en la medida en que se instalan más deflectores. Esta condición también es cierta para la remoción de coliformes, si se tiene en cuenta que los deflectores disminuyen las oportunidades de mezcla y aproximan el flujo al flujo ideal.

\section{DISEÑO DE ESTRUCTURAS}

En el diseño de lagunas de estabilización es indispensable obtener un vaso con la mayor impermeabilidad posible, reducir las pérdidas por infiltración y proteger el agua subterránea de la contaminación. Es necesario excavar suelo y construir diques de poca altura; la construcción de diques exige de estudios de suelos para que no haya hundimientos, no se produzca infiltración ni deslizamiento del talud. La corona de los diques tiene un ancho de 1,50 a 3 metros y los taludes una inclinación variable, en función de la estabilidad del suelo, generalmente de $1: 1$ a $1: 3$, $\mathrm{V}: \mathrm{H}$.

La prueba de infiltración simple permite estimar la pérdida por infiltración, esta prueba se describe en la literatura. Una vez se conocen las pérdidas por infiltración y evaporación se debe hacer un balance hídrico para establecer si es posible llenar el vaso, es decir, conocer si el aporte de agua es mayor a las pérdidas, de otra manera es necesario modificar el diseño.

Se debe tener en cuenta que las dimensiones fijadas del cálculo del área de la laguna corresponden a las del área a la profundidad media. Es así como es necesario tener en cuenta la inclinación de los taludes y el borde libre para encontrar las dimensiones en la corona del dique. La altura del borde libre se calcula mediante la expresión:

$$
B L=[\log A]^{/ 2}-1
$$

Donde : $\mathrm{BL}=$ Borde libre, $\mathrm{m}$

$$
\text { A }=\text { Area de la laguna en metros }
$$
Cuadrados

En muchos casos es necesario impermeabilizar el interior del vaso de la laguna con materiales, tales como arcilla, tierra cemento, concreto o geotextil, el material seleccionado depende del costo, la disponibilidad en el sitio y la complejidad de la instalación.

Otras estructuras necesarias en lagunas de estabilización son: rejas para remover sólidos grandes, desarenadores para separar arena e 
inertes, canales para el transporte de agua en tratamiento, medidores de flujo y compuertas para interrumpir o restablecer flujo a una laguna, además de deflectores para retener material flotante. En la construcción de estas estructuras se utilizan tuberías y materiales resistentes a la corrosión, el concreto debe ser de alta calidad. El uso de válvulas encarece el diseño y la construcción.

La cara externa de los taludes de los diques se debe proteger con vegetación, la siembra de grama es aconsejable, no debe haber árboles.

\section{OPERACIÓN Y MANTENIMIENTO}

La operación de lagunas sin hacer mantenimiento puede dar origen a molestias ambientales, tales como: la proliferación de mosquitos, malos olores y riesgos a la salud. El mantenimiento satisfactorio de las instalaciones de las lagunas permite: producir un efluente de calidad aceptada, evitar molestias ambientales y evitar el deterioro físico de las instalaciones y equipos. Las tareas de rutina en la operación y el mantenimiento de lagunas incluyen:

* Limpieza de rejas y desarenadores y disposición adecuada de sólidos.

* Lubricación periódica de equipos de operación mecánica o automática en rejas, estaciones de bombeo, verificaciones del funcionamiento $y$ mantenimiento de rutina, cuando exista este tipo de equipos en instalaciones grandes.

* Cortar periódicamente la vegetación en la cara exterior de los taludes y no permitir vegetación en la cara interior de los taludes.

* Retirar la espuma y sólidos que flotan en lagunas facultativas y de maduración. En lagunas anaerobias no se debe retirar el material que flota para aislarla del aire.

* Remover la vegetación emergente en las lagunas y el borde del agua para controlar la proliferación de mosquitos.

* Mantenga registros de los caudales que entran y salen y de la calidad del afluente y efluente, para establecer la eficiencia.
* Revise periódicamente si ha habido daño a los diques por la acción de: roedores, serpientes, hormigas, etc. Reconstruya los diques y cree un ambiente hostil a los animales intrusos.

* Dé instrucciones al personal de operación y mantenimiento sobre sus responsabilidades y haga que dejen un registro escrito de sus actividades.

* La limpieza o extracción del lodo acumulado en la laguna se debe hacer cuando llega a la mitad, la profundidad líquida mínima aceptada es de un metro en lagunas facultativas. El lodo se debe retirar cada 5 años en lagunas anaerobias y cada 20 años en lagunas facultativas.

* Ejecute las reparaciones necesarias en la cerca, la iluminación y los taludes de los diques.

\section{Ejercicio}

Una población tiene ahora 13.000 habitantes y se espera que dentro de 20 años tenga 20.000, la producción de aguas residuales es de 190 L/hab.día, la DBO de las aguas residuales es de $215 \mathrm{~g} / \mathrm{m} 3$, la temperatura media del mes más frío del año es de $26^{\circ} \mathrm{C}$, la evaporación media es de 0,005 m/d y la infiltración llega a 0,005 m/d. Se debe hacer un diseño preliminar del sistema de lagunas para un MMP en el efluente igual o menor a 10.000 por $100 \mathrm{ml}$ si el MMP en el afluente es de $1.000 .000 / 100 \mathrm{ml}$ y una $\mathrm{DBO}_{\mathrm{e}}$ menor a $30 \mathrm{~g} / \mathrm{m}^{3}$.

Solución:

1.Flujo de aguas residuales:

.Ahora: $Q=0,19 \mathrm{~m}^{3} / \mathrm{h} . \mathrm{d} \times 13.000 \mathrm{hab}=$ $2.470 \mathrm{~m}^{3} / \mathrm{d}$

.2020 : $\quad Q=0,19 \mathrm{m3} / \mathrm{h} . \mathrm{d} \times 20.000 \mathrm{hab}=$ $3.800 \mathrm{~m}^{3} / \mathrm{d}$

2.Cálculo de la carga orgánica para 20.000 habitantes:

C.O $=3800 \mathrm{~m}^{3} / \mathrm{d} \times 0,215 \mathrm{~kg} / \mathrm{m}^{3}=817$

Kg.DBO/día 
Diseño preliminar de un sistema de lagunas facultativas y de maduración:

Se fija una profundidad de la laguna primaria de 1,80 metros

3. Carga superficial máxima que puede aceptar la laguna facultativa:

$\mathrm{CS}_{\mathrm{m}}=714,28 \times \mathrm{H} \times 1,085^{(\mathrm{T}-35)}=714,28 \times 1,8 \times$ $1,085^{(26-35)}=617 \mathrm{kgDBO} / \mathrm{ha} . \mathrm{d}$

$\mathrm{CS}_{\mathrm{m}}=60,3 \times 1,099^{\top}=60,3 \times 1,099^{26}=702$ $\mathrm{KgDBO} / \mathrm{ha} . \mathrm{d}$

4. Cálculo del área de la laguna para $\mathrm{CS}_{\mathrm{m}}=617$ $\mathrm{Kg} / \mathrm{ha} . \mathrm{d}$

$$
A=\frac{C . O}{C S_{m}}=\frac{817 \mathrm{kgDBO} / \mathrm{d}}{617 \mathrm{KgDBO} / \mathrm{ha} . \mathrm{d}}=1,32 \text { has }
$$

5. Volumen de la laguna, $V=1,32$ ha $\times 10.000$ $\mathrm{m}^{2} / \mathrm{ha} \times 1,80 \mathrm{~m}=23.760 \mathrm{~m}^{3}$

6. Pérdidas por evaporación e infiltración: $Q_{p}=$ $(E+I) A$

$Q_{p}=(0,005+0,005) 1,32$ ha $\times 10.000 \mathrm{~m}^{2} / \mathrm{ha}=$ $132 \mathrm{~m}^{3} / \mathrm{d}$

7. Período de retención

$$
P R=\frac{2 V}{Q_{a}+Q_{e}}=\frac{2 \times V m^{3}}{(3800+3668) m^{3} / d}=6,36 \text { dias }
$$

8. Cálculo de la carga superficial removida, CS, de la Ec. 8 (Cubillos)

$S C_{r}=25,13+0,675 \times 617=442 \mathrm{KgDBO} / \mathrm{ha} . \mathrm{d}$

9.Cálculo de la DBO en el efluente de la laguna:

$\mathrm{CS}_{\mathrm{e}}=\left(\mathrm{Q}_{\mathrm{e}} \times \mathrm{DBO}_{\mathrm{e}}\right) / \mathrm{A}$

$\mathrm{CS}_{\mathrm{e}}=\mathrm{CS}_{\mathrm{m}} \mathrm{CS}_{\mathrm{r}}=617442=175 \mathrm{kgDBO} / \mathrm{ha} . \mathrm{d}$

$D B O_{e}=\frac{175 \mathrm{kgDBO} / \mathrm{ha} \cdot \mathrm{d} \times 1,32 \mathrm{ha} \times 1000 \mathrm{~g} / \mathrm{kg}}{3668 \mathrm{~m}^{3} / \mathrm{d}}=63 \mathrm{~g} / \mathrm{m}^{3}$
10.Dimensiones de la laguna: selecciono dos lagunas de $L=120 \mathrm{~m}$ y $B=55 \mathrm{~m}$.

11. Cálculo del MMP de coliformes en el efluente para laguna con flujo disperso

$d=\frac{L / B}{-0,26+0,254(L / B)+1,014(L / B)^{2}} \quad($ Yanez $)$

$d=\frac{120 / 55}{-0,261+0,254(120 / 55)+1,014(120 / 55)^{2}}=0,426$

$K_{b}=0,917 H^{-0,877}(P R)^{-0,329}=0,917 \times 1,8^{-0,877} 6,36^{-0,329}=0,30 / d \quad a 20^{\circ} \mathrm{C}$

$K_{T}=K_{20} \Theta^{(T-20)} \quad K_{26}=0,30 \times 1,07^{(26-20)}=0,45 / d$

$d=\frac{L / H}{-0,261+0,254(L / H)+1,014(L / H)^{2}}=\frac{120 / 55}{-0,261+0,254(120 / 55)+1,014(120 / 55)^{2}}=0,426$

$d=\frac{120 / 55}{-0,261+0,254(120 / 55)+1,014(120 / 55)^{2}}=0,426$

$a=\sqrt{1+4 K_{b} x P R x d}=\sqrt{1+4 x 0,45 x 6,36 \times 0,426}=2,42$

$N=\frac{4 a \mathrm{E}^{[(1-a) / 2 d]}}{(1+a)^{2}} N_{0}=\frac{4 x 2,42 x \mathrm{E}^{(1-2,42) / 2 x 0,426}}{(1+2,42)^{2}} \times 10^{6}=156.314$

Es necesario diseñar una laguna secundaria en serie para reducir el MMP de coliformes

\section{Diseño preliminar de la laguna secundaria facultativa}

La DBO total en el afluente se toma igual a dos veces la DBO soluble en el efluente de la laguna primaria.

DBO total $=2 \times 63=126 \mathrm{~g} / \mathrm{m}^{3}$

Carga orgánica que llega: $C O=3668 \mathrm{~m}^{3} / \mathrm{d} x$ $0,126 \mathrm{~kg} / \mathrm{m}^{3}=462 \mathrm{~kg} / \mathrm{d}$

La carga superficial máxima se fija de la experiencia en $400 \mathrm{~kg} / \mathrm{ha}$. d para este caso.

Area de la laguna, $A=C O / S C_{m}=(462 \mathrm{~kg} / \mathrm{d}) /$ $(400 \mathrm{Kg} / \mathrm{ha} . d)=1,16 \mathrm{ha}$

Profundidad de la laguna $=1,80 \mathrm{~m}$. 
Volumen de la laguna $=\mathrm{V}=1,16$ ha $\times 10.000$ $\mathrm{m}^{2} / \mathrm{ha} \times 1,8 \mathrm{~m}=20.880 \mathrm{~m}^{3}$

Pérdidas por evaporación más infiltración $=0,01$ $\times 11.600 \mathrm{~m}^{2}=116 \mathrm{~m}^{3} / \mathrm{d}$

Período de retención, $P R=(2 \times 20.880) /$ $(3668+3552)=5,8$ días

$\mathrm{CS}_{\mathrm{e}}=0,8167 \times \mathrm{CS}_{\mathrm{m}} \quad 3,82=0,8167 \times 4003,82$ $=323 \mathrm{~kg} / \mathrm{ha} . \mathrm{d}$

Carga superficial en el efluente, CS $_{\mathrm{e}}=400323$ $=77 \mathrm{~kg} / \mathrm{ha} . \mathrm{d}$

$\mathrm{DBO}_{\mathrm{e}}$ en el efluente $=\left(\mathrm{CS}_{\mathrm{e}} \times \mathrm{A}\right) / \mathrm{Q}_{\mathrm{e}}=(77 \times 1,16 \times$ 1000) $/ 3552=25 \mathrm{gDBO} / \mathrm{m}^{3}$

Dimensiones de la laguna: dos lagunas de $\mathrm{L}=$ $105 \mathrm{~m}, \mathrm{~B}=55 \mathrm{~m}, \mathrm{H}=1,80 \mathrm{~m}$

\section{Cálculo del MMP en el efluente de la segunda laguna}

$K_{b}=0,917 \times 1,8^{-0,877} \times 5,8^{-0,329}=0,307 / d$

$K_{b(26)}=0 ; 307 \times 1,07^{(26-20)}=0,46 / d$

$d=\frac{105 / 55}{-0,261+0,254(105 / 55)+1,014(105 / 55)^{2}}=0,487$

$a=\sqrt{1+4 x K_{b} x P R x d}=\sqrt{1+4 x 0,46 \times 5,8 x 0,487}=2,48$

$N=\frac{4 a \mathrm{E}^{(1-a) / 2 d} N_{1}}{(1+a)^{2}}=\frac{4 x 2,48 x \mathrm{E}^{(1-3,48) / 2 x 0,487} x 156.314}{(1+2,48)^{2}}=28.018$

Es necesario diseñar una tercera laguna en serie con las lagunas anteriores, esta laguna es de maduración porque se diseña para remover coliformes y recibe aguas tratadas.

\section{Diseño preliminar de la laguna de maduración}

Se diseñan dos lagunas de 55 metros de ancho, 1,0 metros de profundidad y 70 metros de longitud.
Volumen de la laguna: $\mathrm{V}=2(55 \times 70 \times 1,0)=$ $7.700 \mathrm{~m}^{3}$

Pérdidas por evaporación e infiltración $=7.700$ $\mathrm{m}^{2} \times 0,01 \mathrm{~m} / \mathrm{d}=77 \mathrm{~m}^{3} / \mathrm{d}$

Período de retención: $P R=2 \times 7.700 /$ $(3.552+3.475)=2,19 d$

$$
\begin{gathered}
K_{b}=0,917 \times 1,0^{-0,877} \times 2,19^{-0,329}=0,71 / d \\
K_{b(26)}=0,71 x 1,07^{(26-20)}=1,06 / d
\end{gathered}
$$

$$
\begin{gathered}
d=\frac{70 / 55}{-0,261+0,254(70 / 55)+1,014(70 / 55)^{2}}=0,746 \\
a=\sqrt{1+4 \times 1,06 \times 2,19 \times 0,746}=2,72
\end{gathered}
$$

$N=\frac{4 \times 2,72 \times \mathrm{E}^{(1-2,72) / 2 \times 0,746} \times 28.018}{(1+2,72)^{2}}=6.955 / 100 \mathrm{ml}$

Esta cifra satisface las exigencias para el diseño de obtener un efluente con menos de 10.000 coliformes por $100 \mathrm{ml}$ -

\section{REFEREMCIAS}

1.Cubillos J.A. 1984 "Criterios para el Dimensionamiento de Lagunas de Estabilización" CIDIAT, Mérida, Venezuela.

2. Kawai H. 1981 "Descricao das Avalicoes de Lagoas de Estabilizacao no Brasil" Lima, Moviembre. Curso sobre Lagunas de Estabilización en CEPIS.

3. Kilani J.S. y Ogunrombi J.A. 1988 "Effects of Baffles on the Performance of Model Waste Stabilization Ponds" Water Res. Vol.18 Mo 8, p941-944.

4.León G. y Moscoso J., 1996. "Curso de Tratamiento y Uso de Aguas Residuales" OPS/OMS/PUB96.20, Lima, Perú.

5. Mara D.D. y Silva S. A. 1969 "Tratamientos Biológicos de Aguas Residuarias, Lagoa de 
Stabilizacao"Associacao Brasileira de Engenharia Sanitaria.

6. Marais G.V.R. 1966 "New Factors in the Design, Operation and Performance of Waste Stabilization Ponds"Bull WHO, 34, p737.

7. Marais G.V.R. 1974 "Faecal Bacterial Kinetics in Stabilization Ponds" Jour. Env. Eng. ASCE, Feb. (p119-139).

8. Mc Garry M.G. y Pescod M.B, 1970 "Stabilization Pond Design Criteria for Tropical Asia" Second International Symposium for Waste Treatment Lagoons. June 23-25, KansasCity.

9. Oswald W.J y Gotaas H.B. 1957 "Photosynthesis in Sewage Treatment" Trans. ASCE, V.122

10. Oswald W.J. 1995 "Ponds in the Twenty First Century" Wat, Sci Tech vol 31. Mo.12.

11.Pearson H.W. 1995 "Expanding the Horizons of Pond Technology and Application in an Environmentally Conscious World" Wat. Sci. Tech. Vol.33. Mo 7 (p.1-9).

12.Polprasert C y Bhattarai K.K. 1985 "Dispersion Model for Waste Stabilization Ponds" Jour Env. Eng, Vol 111 Mo 1, ASCE (p 45 - 59).

13.Romero J.A. 1995 "Acuitratamiento por Lagunas de Estabilización" Ecsuela Colombiana de Ingeniería, Santa Fé de Bogotá, Colombia

14.Thirumurthi A.M.1969 "Design Principles of Waste Stabilization Ponds"Jour San Eng Div ASCE, Vol.95, Mo SA2, p.311-330.

15.Von Sperling M, 1999, "Performance Evaluation and Mathematical Modeling of Coliform Die-off in Tropical and subtropical Waste Stabilization Ponds" Water Research. Vol 33 PP 1435.
Waste Stabilization Ponds" Water Research. Vol 33 pp 1435. Elsevier Science Ltd.

16.Yanez F, 1980 "Lagunas Facultativas y de Alta Producción de Biomasa" CEPIS, Lima, Perú.

17.Yanez F, 1993 "Lagunas de Estabilización, Teoría, Diseño, Evaluación y Mantenimiento" Empresa Pública de Teléfonos, Agua Potable y Alcantarillado de Cuenca. Casilla 297, Cuenca, Ecuador. 